\title{
Defects Generation in Single-Walled Carbon Nanotubes Induced by Soft X-ray Illumination
}

\author{
Yutaka Mera*a, Yoshihisa Harada ${ }^{b}$, Satoshi Arimaa, Kenji Hatad, Shik \\ Shin ${ }^{c}$, Koji Maedaa \\ aDept. of Applied Physics, The University of Tokyo, Tokyo 113-8656 Japan \\ bDept. of Applied Chemistry, The University of Tokyo, Tokyo 113-8656 \\ Japan \\ cISSP, The University of Tokyo, Chiba 277-8581 Japan \\ dNational Institute of Advanced Industrial Science and Technology, Ibaraki \\ Japan
}

\begin{abstract}
Defects generation in single-walled carbon nanotubes induced by soft X-rays from a synchrotron radiation source was investigated by in-situ C 1s soft X-ray absorption and recombination emission as well as ex-situ micro-Raman scattering spectra. The spectral dependence of the defect-generation efficiency shows that the illumination effect is resonantly enhanced in between the $\pi^{*}$ and $\sigma^{*}$ carbon 1 s core absorption. A spectator Auger decay process is proposed as the most plausible mechanism for the defects generation.
\end{abstract}

\section{Keywords}

Carbon Nanotubes; Defect; Core Excitation; Soft X-ray Spectroscopy; Syncrotron Radiation

*Corresponding Author: Yutaka Mera

Dept. of Applied Physics, School of Engineering, The University of Tokyo, 7-3-1, Hongo, Bunkyo-ku, Tokyo 113-8656 Japan

Email: mera@exp.t.u-tokyo.ac.jp Phone \& Fax: +81-3-5841-6852 
Single-walled carbon nanotubes (SWNTs) have unique properties quite variable depending on their structures. Particularly, the electronic properties of SWNTs vary from metallic to semiconducting according to with what helicity and a diameter the graphene sheet is folded. Although electrons in SWNTs retain high mobility even in the presence of defects and impurities due to the lack of high angle scattering in the one-dimensional structures ${ }^{1}$, the electronic transport and the electronic structures would be still significantly affected by the presence of defects. An example is the metal-semiconductor transition induced by electron irradiation that was found in metallic nanotube devices which change their behavior as single-electron transistors to the behavior as ambipolar field-effect transistors after exposed to an electron beam of 1 $\mathrm{keV}^{2}$. Similarly, higher energy $(20 \mathrm{keV})$ electron irradiation causes a drastic decrease of the electric conductivity for both metallic and semiconducting nanotubes ${ }^{3}$. Attempts to fabricate single quantum dots on carbon nanotubes with tunnel barriers formed by argon-ion irradiation ${ }^{4}$ suffer from poor reproducibility in the barrier height due to the lack of knowledge about the nature of the radiation-induced defects. Hence, to realize nanotube-based devices on a sound basis, it is crucial to have a well-founded knowledge of the defects in nanotubes.

For carbon materials, it is known that excitations of carbon $1 \mathrm{~s}(\mathrm{C} 1 \mathrm{~s})$ core electrons by soft X-ray illumination cause momentary atomic displacements in crystalline diamond and graphite ${ }^{5,6}$ and irreversible structural changes in amorphous carbon solids ${ }^{7}$. More recently, it has become realized that light illumination introduces defects also in SWNTs. The intensity of defect-related D-band in Raman spectra was found to increase after illumination with a synchrotron radiation $(20 \mathrm{eV})^{8}$. Unknown midgap luminescence centers in SWNTs were reported to be generated by light illumination at a lower energy of $6 \mathrm{eV}$ which electronically excites surfactant molecules attached to the SWNTs ${ }^{9}$.

In this letter, we report a generation of defects in SWNTs induced by soft X-ray illumination exciting $\mathrm{C}$ 1s core electrons, which is detected by X-ray absorption spectroscopy (XAS) and optical Raman spectroscopy. Possible mechanisms are discussed for the microscopic processes after the core excitation.

The SWNT samples used in this study were synthesized by the Super Growth Method ${ }^{10}$, chemical vapor deposition under a small amount of water vapor for an efficient reactivator of the metal catalyst. The average diameter and the length of the tubes were $2 \mathrm{~nm}$ and over a few hundred microns, respectively. The SWNTs were dispersed in dimethyl formamide (DMF) and then were deposited onto Si substrates with a syringe.

The soft X-ray illumination and XAS measurements were conducted at room 
temperature in a high vacuum of $5 \times 10^{-8} \mathrm{~Pa}$ in the beam-line BL27SU at SPring-811. In order to induce a structural change in the SWNT films, we used intense soft X-rays

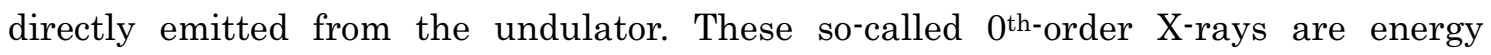
variable and nearly monochromatic with the full width at half maximum (FWHM) of about $7 \mathrm{eV}$ near the $\mathrm{C} 1 \mathrm{~s}$ absorption edge. The instruments are designed to stabilize the size and the position of the X-ray beam at the focal point during the intense

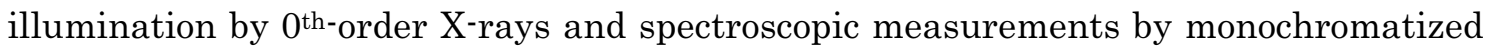
$\mathrm{X}$-rays. The vertical size of the X-ray beam measured by knife-edge scanning was $20 \mathrm{~m}$ at the focal point.

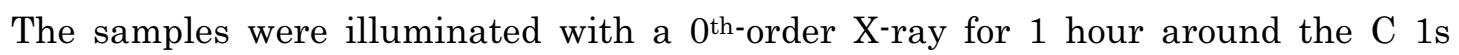

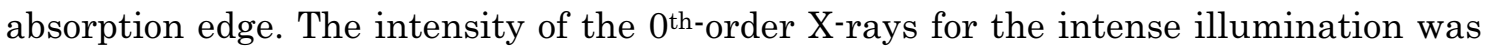
adjusted at $10^{18}$ photons $/\left(\mathrm{s} \cdot \mathrm{cm}^{2}\right)$, more than two orders of magnitude higher than the flux density of incident photons used for the spectroscopic measurements. The center of the photon energy for this illumination was tuned from $270 \mathrm{eV}$ to $305 \mathrm{eV}$. Before and after each X-ray illumination XAS spectra in the total electron yield (TEY) mode were acquired by measuring the sample drain current. Then we subtracted the XAS spectra in order to see structural changes induced in the SWNT films by the intense X-ray illumination.

In order to study an effect on the defect generation, i.e. the effect of a neutral (core-excitonic) excitation without a valence hole, we observed C 1s recombination emission. As discussed later, the recombination emission is sensitive to an ultrafast $(\sim 10 \mathrm{fs})$ dissociation of the excited atom just after the resonant core excitation ${ }^{5,6}$ which might initiate a defect generation. The recombination emission spectra were measured for the sample before the soft X-ray illumination by using a flat field soft X-ray emission spectrometer ${ }^{12}$. The energy resolution of the XES spectrometer was better than $0.6 \mathrm{eV}$ at the $\mathrm{C}$ 1s edge. The incident photon energies for these spectroscopic measurements were calibrated using the energy position of $\mathrm{Au}$ 4f photoemission lines.

After the soft X-ray illumination experiments, the sample was examined ex-situ by micro Raman spectroscopy using a Renishaw inVia Raman microscope with an excitation laser of $532 \mathrm{~nm}$ in wavelength to detect the spatial correlation of Raman spectral changes to the soft X-ray illumination. The perfectness of SWNTs was assessed by the so-called G/D ratio, the ratio of the integral intensity of the G-band peaking at $1582 \mathrm{~cm}^{-1}$, which represents the graphene walls in SWNTs, to that of the D-band, the broad spectrum around $1336 \mathrm{~cm}^{-1}$ that indicates the presence of defects in the graphene walls. The integral intensity was calculated in the range of $1497 \mathrm{~cm}^{-1}$ to $1667 \mathrm{~cm}^{-1}$ for the G-band and $1251 \mathrm{~cm}^{-1}$ to $1421 \mathrm{~cm}^{-1}$ for the $\mathrm{D}$-band. The spatial resolution of the 
microscopic measurements was $5 \mathrm{~m}$ under the just-focus condition.

Figure 1 shows XAS-TEY spectra measured before and after illumination for $60 \mathrm{~min}$ at $284.8 \mathrm{eV}$. The normalized spectra appear almost indistinguishable with naked eyes but there exists a subtle difference. The dotted line shows the illumination-induced change from the initial spectrum to the spectrum after illumination. The intensity increase at $284 \mathrm{eV}$ and $286 \mathrm{eV}$ and a decrease at $285 \mathrm{eV}$ in the dotted curve indicate a broadening of the $285 \mathrm{eV}$ XAS peak that is assigned to optical transitions from the $\mathrm{C} 1 \mathrm{~s}$ core level to $\mathrm{C}-\mathrm{C} \pi^{*}$ states originating in the $\mathrm{sp}^{2}$ hybrid bonds of carbon atoms in the graphitic sheets of SWNTs.

To quantify the degree of the peak broadening, we calculated the integral breadth $w$ of the peak that is defined by the width of a rectangle with an area and a height both of which are the same as the peak ${ }^{13}$. We further introduced a quantity named $\pi^{*}$ peak broadening $B \equiv\left(w_{f}-w_{i}\right) / w_{i} / P$, where $w_{i}$ and $w_{f}$ are the breadth in the initial spectrum and that after illumination, respectively, and $P$ the photon fluence of the $0^{\text {th-order light }}$ calculated from the experimentally measured sample current and illumination time. The solid circles in Figure 2 shows the excitation spectrum for the $\pi^{*}$ peak broadening obtained by plotting $B$ as a function of the photon energy used for the intense illumination. The spectrum has a resonant structure peaking at an energy around 289 $\mathrm{eV}$ with a non-resonant background near the $\mathrm{C} 1 \mathrm{~s}$ core absorption edge.

Figure 3 shows the results of the $\mathrm{C} 1$ s recombination emission measurements. At all excitation energies, the emission spectra showed quite symmetric profile. peaking at the excitation energy(elastic peak), This is in contrast to the cases of diamond( $\left.\mathrm{sp}^{3}\right)$ and graphite $\left(\mathrm{sp}^{2}\right)$ where the recombination emission spectra across the core exciton excitation exhibited intense low-energy tail ${ }^{5,6}$.

The solid and broken curves in Fig. 4(a) indicate typical Raman spectra measured at two positions on the SWNT sample illuminated with intense soft X-rays (red dashed line) and not illuminated (blue line), respectively. Figure 4(b) shows the spatial distribution of inverse G/D ratio over the sample. The eight stripes in bright contrasts corresponding to the illuminated areas indicate that defects were introduced by the soft X-ray illuminations. Figure 4(c) shows a profile of inverse G/D ratio along the white line in Fig.4(b) where the sample was best focused. The Gaussian fitting curve with the FWHM of $19.7 \mathrm{~m}$ agrees well with the beam profile of the soft X-rays use for intense illumination. The open marks in Figure 2 show the dependence on the illumination-photon energy of the degree of G/D degradation in the micro Raman spectra. The G/D degradation spectrum also has a resonant structure peaking at an energy around $289 \mathrm{eV}$, but it has a substantial non-resonant component in contrast to 
the XAS peak broadening spectrum.

Generally, defects or disordered structures cause an inhomogeneous broadening in optical transition spectra. As discussed on broadened XAS peaks of multi-walled carbon nanotubes ${ }^{14}$, it may be reasonable to consider that the $\pi^{*}$ peak broadening in XAS is attributed to the generation of defects also in the SWNTs. The defect generation in SWNTs by light illumination in the soft X-ray wavelength is clearly evidenced by the Raman experiment as shown in Fig. 4(b). As evidenced by the two excitation spectra in Fig. 2, the illumination effect is enhanced in a resonant manner at photon energies in a range above the $\mathrm{C} 1 \mathrm{~s}$ core absorption edge. Another change noticed in the XAS-TEY spectra (Fig. 1) is an increase of the spectral intensity near $290.5 \mathrm{eV}$. The $290.5 \mathrm{eV}$ peak, which is not visible in the XAS spectra but is detected as a photo-induced signal, was attributed to defects in MWNTs ${ }^{15}$ and graphite ${ }^{16}$.

One mechanism which is usually invoked to explain atomic movements induced by resonant core excitations is the bond instability in a spectator-Auger final state ${ }^{17,18}$. In this mechanism, the electron core-excited to an unoccupied upper level of anti-bonding nature stays at the level spectating the Auger decay that generates two or more holes in lower valence levels, the latter of which could cause Coulomb explosion, an explosive repulsion of atoms by imbalanced Coulomb forces ${ }^{19}$. Only for two- or multiple-hole states to be generated, it occurs also in normal Auger processes, in which a core electron is non-resonantly excited above the vacuum level and subsequently the emptied core level is filled by a valence electron via Auger decay generating multiple holes in the valence levels. The important difference of the spectator Auger final state and the final state of normal Auger decay is the presence of the spectator electron at the upper anti-bonding level in the former case which should more strongly enhance the lattice instability due to Coulomb explosion induced by the multiple holes. Since the non-resonant normal Auger processes are induced by illumination at $305 \mathrm{eV}$, the experimentally observed reduction of the illumination effect at such a high energy, as shown in Fig. 2, strongly rules out the normal Auger mechanism for the defects generation. For carbon nanotubes, Miyamoto et al. showed theoretically that core excitations of oxygen $1 \mathrm{~s}$ core electrons induce the desorption of the oxygen atoms as a consequence of the formation of two holes in the valence $\mathrm{O} 2 \mathrm{~s}$ states and the occupation of $\mathrm{O} 2 \mathrm{p}-\mathrm{C} 2 \mathrm{p}$ hybrid states of anti-bonding character ${ }^{20}$. These results suggest that a spectator Auger mechanism is responsible for the resonantly induced defect formation in the present study.

Another conceivable mechanisms of such resonant core excitation effects is the pseudo-Jahn-Teller distortion in the core exciton state (the electron excited from a C $1 \mathrm{~s}$ 
level being bound by the Coulomb force to the generated core hole) proposed first by $\mathrm{Ma}^{5}$. It was shown that the recombination emission spectrum induced by a resonant core excitation exhibits a peculiar long low-energy tail in addition to a small Stokes shift when the $\mathrm{C} 1$ s core electron is excited into the core exciton state in diamond ${ }^{5}$ and in graphite $^{5,6}$. The presence of such a phonon sideband in the hot luminescence indicates the occurrence of considerable atomic displacements within the short lifetime of the core exciton state. Theoretical calculations ${ }^{21-23}$ showed that the symmetry-breaking pseudo-Jahn-Teller (PJT) distortion mechanism operates in the core exciton state causing a temporal displacement of carbon atoms from the lattice position in high symmetry. In the present phenomenon, however, no phonon sideband was observed as shown in Fig. 3. If the defect generation takes place at normal C sites in SWNT, the absence of phonon sideband rules out the core-exciton mechanism for the resonantly induced damage effect. However, if the defect generation takes place at some specific sites small in number, it should be difficult to detect the phonon sideband due the PJT mechanism that operates at the minor sites ${ }^{24}$. This problem is related to what follows.

The question remaining is on the origin of the $289 \mathrm{eV}$ peak in the excitation spectrum. There are some studies on the origin of the fine structures in XAS spectra of SWNTs in the photon energy range of 288-292 eV. Kuznetsova et al. attributed the XAS peaks ranging in $287-289 \mathrm{eV}$ to $\mathrm{C}=\mathrm{O} \pi^{*}$ resonances and the peaks in $289-291 \mathrm{eV}$ to $\mathrm{C}=\mathrm{O} \sigma^{*}$ resonances, both associated with oxygen-containing functional groups such as carboxylate ${ }^{25}$. Tang et al. assigned a prominent $289 \mathrm{eV}$ peak observed in their MWNTs to $\mathrm{C}-\mathrm{H} \sigma^{*}$ bonds ${ }^{15}$. For amorphous carbon films, the assignment of $289 \mathrm{eV}$ peak to $\mathrm{C}-\mathrm{H}$ $\sigma^{*}$ resonances has been claimed by many groups ${ }^{26-28}$. Although the XAS-TEY spectra in the present sample exhibited no signal at $289 \mathrm{eV}$ (Fig. 1), the excitation spectrum (Fig. 2) yielded a peak at $290.5 \mathrm{eV}$ close to that energy position. Since it is quite likely that the present SWNT sample was contaminated or decorated with such chemical species, and, whichever the case, $\mathrm{C}=\mathrm{O} \pi^{*}$ or $\mathrm{C}-\mathrm{H} \sigma^{*}$, the resonant excitation of a core electron to the empty level is antibonding in nature, it is reasonable to expect the spectator Auger mechanism to operate inducing bond breaking to form a defect near the chemically modified carbon. This model is consistent with the absence of phonon sidebands in REXS spectra (Fig. 3).

The similar resonant structure in the damage excitation spectra (Fig. 2) between the broadening in XAS spectra and the G/D degradation in Raman spectra suggests that the mechanisms of the two effects are the same. The non-resonant component significant in the G/D degradation spectrum may be explained by the secondary electron effects, which might share a common mechanism with the degradation of G/D ratio induced by 
low-energy $(6 \mathrm{eV})$ photon irradiation. ${ }^{29}$

We investigated the structural change in SWNTs induced by core-electron-exciting soft X-ray illumination by conducting measurements of X-ray absorption, X-ray recombination emission and micro Raman spectroscopy. Two-dimensional mapping of Raman G/D ratio, a measure of structural perfection, indicated that some defects were generated in the SWNTs sample by intense soft X-ray illumination. The defect generation, quantified by broadening of the $\mathrm{C}-\mathrm{C} \pi^{*}$ peak at $285 \mathrm{eV}$ and the degree of G/D degradation, was resonantly enhanced at a photon energy near $289 \mathrm{eV}$. A spectator Auger mechanism was proposed for the defect generation by the resonant core excitations.

We are grateful to the staff of the SPring-8 facility for their assistance in the experiments. The authors also thank Dr. Matthew Bloomfield (Renishaw plc) for the micro Raman measurements. This work was supported by the Ministry of Education, Science, Sports and Culture through Grant-in-Aids for Scientific Research (No. 17340097, No. 19651050, No. 17201023). One of the authors (Y.H.) is grateful to JST/CREST for financial support. 
Figure Captions

Fig.1

XAS-TEY spectra before and after illumination at $284.8 \mathrm{eV}$. The broken line indicates the difference between the initial spectrum and the spectrum after illumination.

Fig. 2

The excitation spectrum for the photo-induced damage deduced from broadening of $\pi^{*}$ peak in XAS spectra (solid circles) and the degree of G/D degradation in Raman spectra (open circles) with dashed lines as eye guides. The XAS spectrum is also shown for comparison.

Fig. 3

Resonantly excited X-ray emission spectra for several excitation energies (vertical broken lines) characteristic in the typical XAS spectrum shown at the top for comparison. Note the absence of low energy tailing in all the spectra.

Fig.4

(a) Typical Raman spectra measured at the SWNT sample positions that were not illuminated (solid line) and illuminated (dashed line) with an intense soft X-ray at 287.5 eV. (b) Two-dimensional map of inverse G/D ratio showing increase of defects in stripe contrasts each corresponding to the sample area intensely illuminated with a photon energy indicated on the top. (c) A cross sectional profile of inverse G/D ratio along the white line in Fig. 4 (b). The broken line is the Gaussian fitting curve with full width of half maximum of $19.7 \mathrm{~m}$ which agrees with the beam profile of soft X-rays use for the intense illumination. 


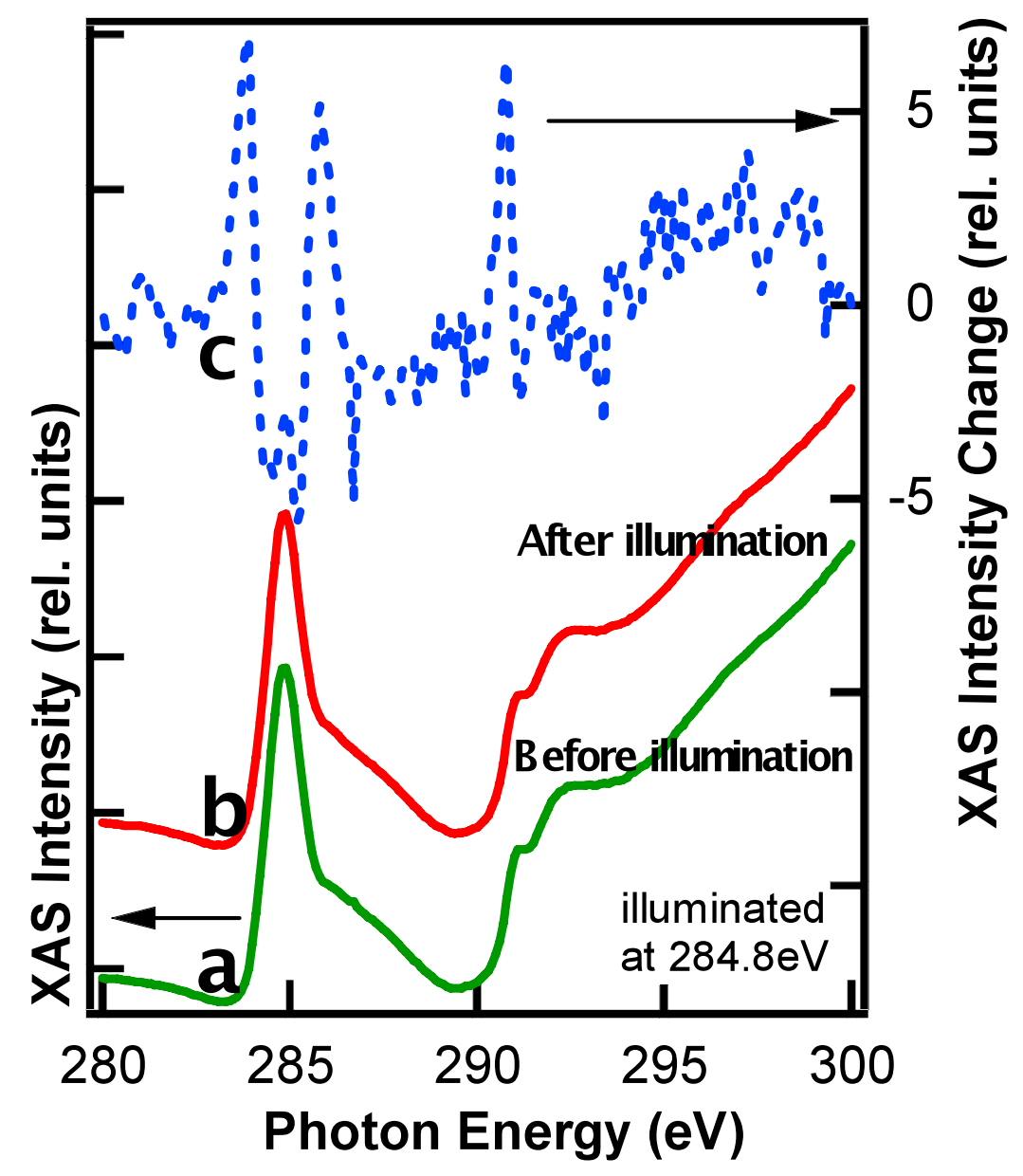

Fig. 1

XAS-TEY spectra before and after illumination at $284.8 \mathrm{eV}$. The broken line indicates the difference between the initial spectrum and the spectrum after illumination. 


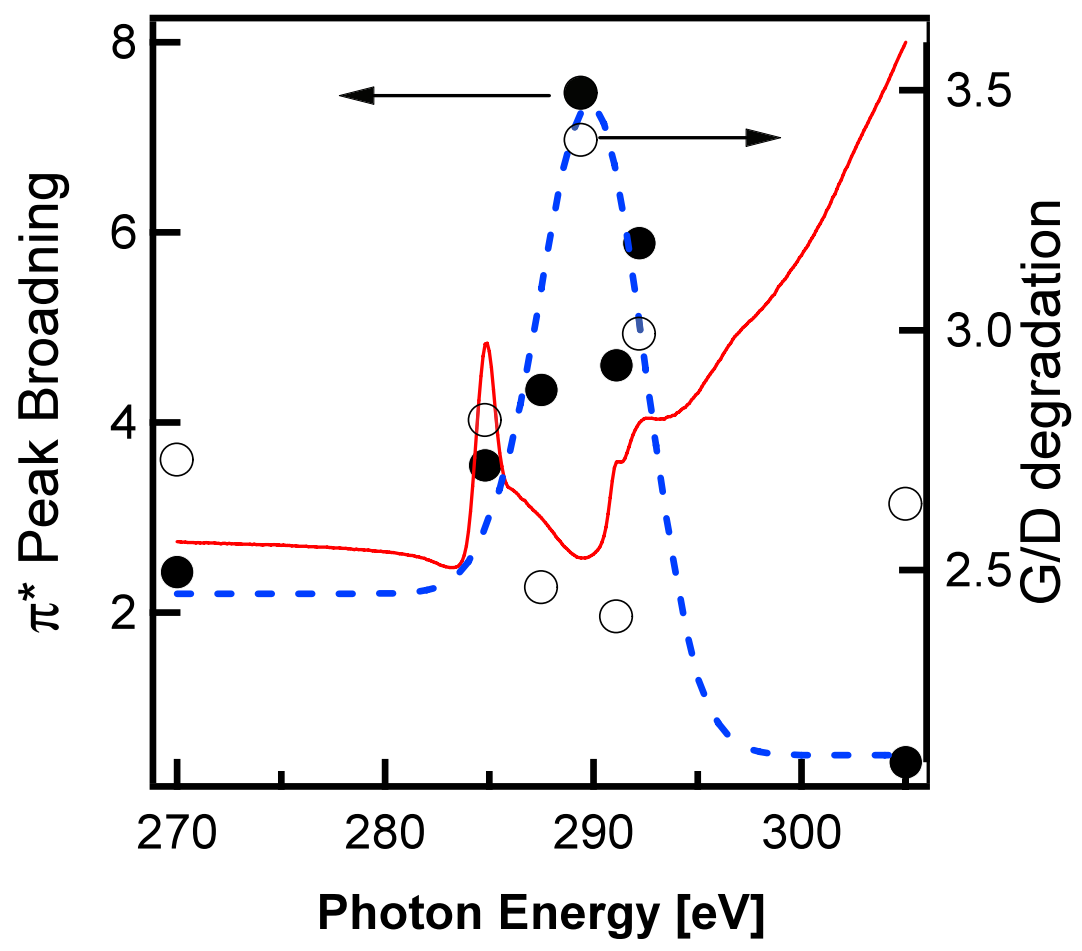

Fig. 2

The excitation spectrum for the photo-induced damage deduced from broadening of $\pi^{*}$ peak in XAS spectra (solid circles) and the degree of G/D degradation in Raman spectra (open circles) with dashed lines as eye guides. The XAS spectrum is also shown for comparison. 


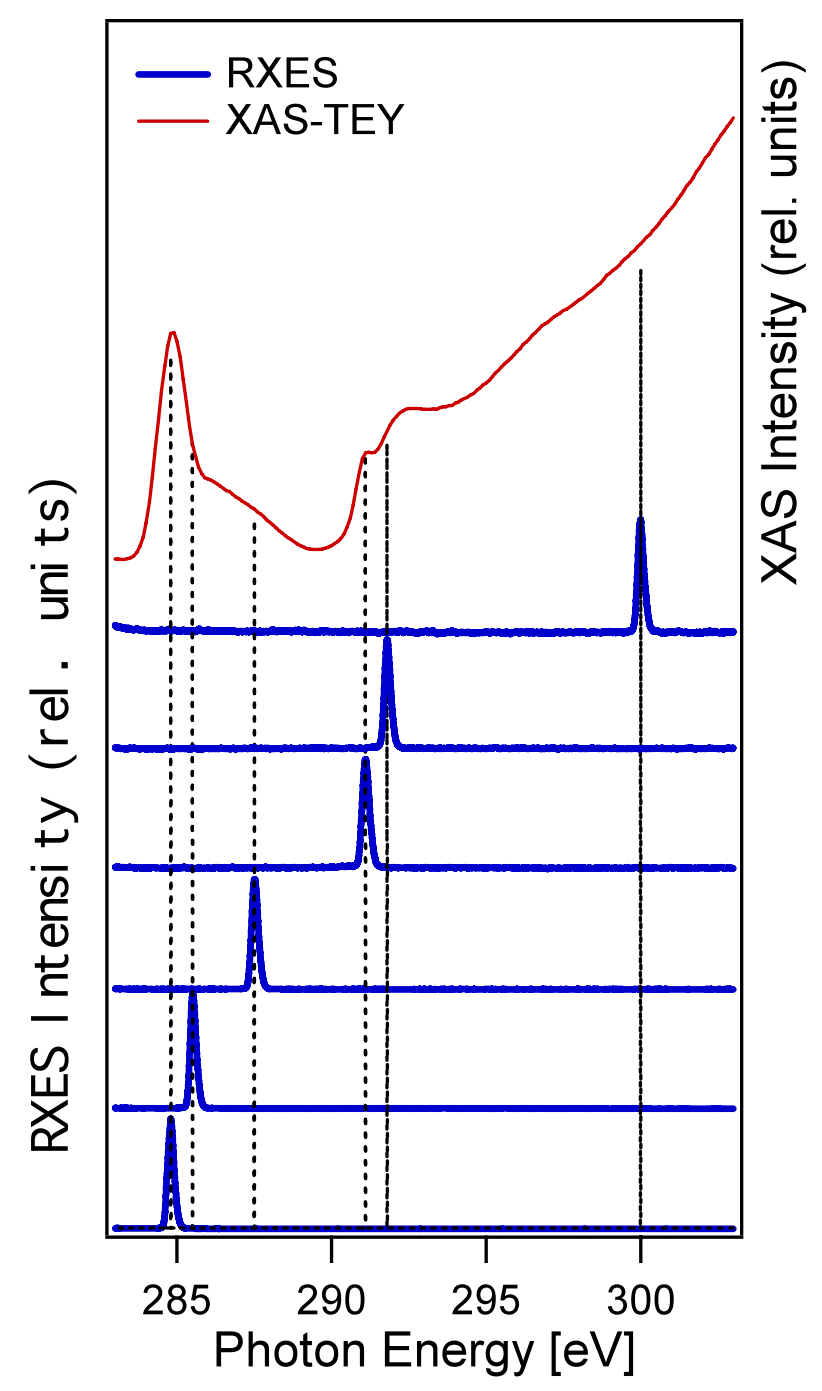

Fig. 3

Resonantly excited X-ray emission spectra for several excitation energies (vertical broken lines) characteristic in the typical XAS spectrum shown at the top for comparison. Note the absence of low energy tailing in all the spectra. 

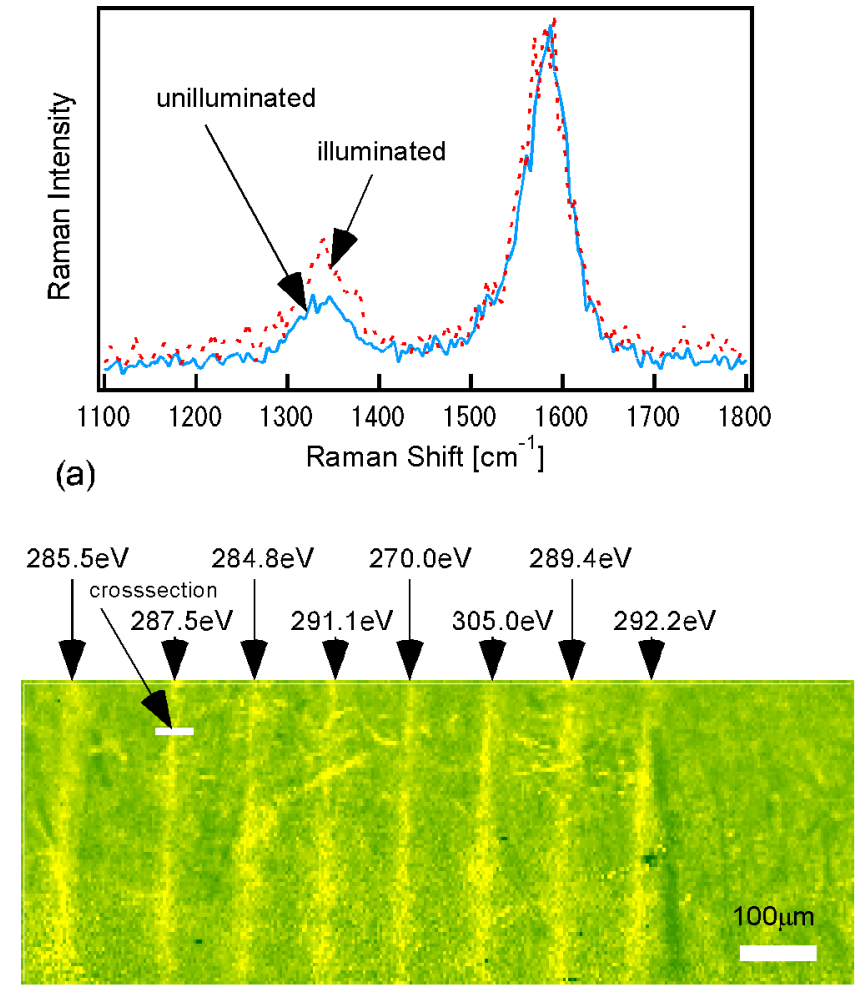

(b)

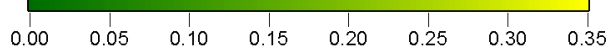

(c)

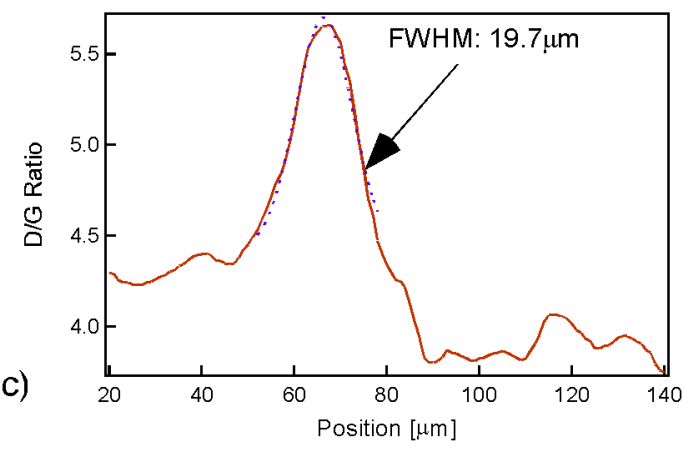

Fig.4

(a) Typical Raman spectra measured at the SWNT sample positions that were not illuminated (solid line) and illuminated (dashed line) with an intense soft X-ray at 287.5 eV. (b) Two-dimensional map of inverse G/D ratio showing increase of defects in stripe contrasts each corresponding to the sample area intensely illuminated with a photon energy indicated on the top. (c) A cross sectional profile of inverse G/D ratio along the white line in Fig. 4 (b). The broken line is the Gaussian fitting curve with full width of half maximum of $19.7 \mathrm{~m}$ which agrees with the beam profile of soft X-rays use for the intense illumination. 
References

[1] T. Ando, T. Nakanishi, J. Phys. Soc. Jpn. 67 (1998) 1704.

[2] A. Vijayaraghavan, K. Kanzaki, S. Suzuki, Y. Kobayashi, H. Inokawa, Y. Ono, S. Kar, P. M. Ajayan, Nano Lett. 5 (2005) 1575.

[3] S. Suzuki, Y. Kobayashi, Jap. J. Appl. Phys. 44 (2005) L1498.

[4] M. Suzuki, K. Ishibashi, K. Toratani, D. Tsuya, Y. Aoyagi, Appl. Phys. Lett. 81 (2002) 2273.

[5] Y. Ma, P. Skytt, N. Wassdahl, P. Glans, J. Guo, J. Nordgren, Phys. Rev. Lett. 71 (1993) 3725.

[6] Y. Harada, T. Tokushima, Y. Takata, T. Takeuchi, Y. Kitajima, S. Tanaka, Y. Kayanuma, S. Shin, Phys. Rev. Lett. 93 (1993) 017401.

[7] S. Liang, Y. Harada, S. Shin, Y. Kitajima, Y. Mera, K. Maeda, Jpn. J. Appl. Phys. 44 (2005) L1472.

[8] S. Suzuki, Y. Kobayashi, Chem. Phys. Lett. 430 (2006) 370.

[9] K. Iakoubovskii, N. Minami, Y. Kim, K. Miyashita, S. Kazaoui, B. Nalini, Appl. Phys. Lett. 89 (2006) 173108.

[10] K. Hata, D. N. Futaba, K. Mizuno, T. Namai, M. Yumura, S. Iijima, Science 306 (2004) 1362.

[11] T. Tanaka, H. Kitamura, J. Synchrotron Rad. 3 (1996) 47.

[12] T. Tokushima, Y. Harada, M. Watanabe, Y. Takata, E. Ishiguro, A. Hiraya, S. Shin, Surf. Rev. Lett. 9 (2002) 503.

[13] R. L. Snyder, J. Fiala, H. J. Bunge, Defect and Microstructure Analysis by Diffraction, Oxford Univ. Press, New York, 1999.

[14] J. Schiessling, L. Kjeldgaard, F. Rohmund, L. K. L. Falk, E. E. B. Campbell, J. Nordgren, P. A. 
Bruhwiler, J. Phys.: Condens. Matter 15 (2003) 6563.

[15] Y. H. Tang, T. K. Sham, Y. F. Hu, C.S. Lee, S. T. Lee, Chem. Phys. Lett. 366 (2002) 636.

[16] J. C. Anguss, C. C. Hayman, Science, 241 (1988) 913.

[17] W. Eberhardt, T. K. Sham, R. Carr, S. Krummacher, M. Strongin, S. L. Weng, D. Wesner, Phys. Rev. Lett. 50 (1983) 1038.

[18] K. Mase, M. Nagasono, S. Tanaka, T. Urisu, E. Ikenaga, T. Sekitani, K. Tanaka, J. Chem. Phys. 108 (1998) 6550.

[19] P. J. Feibelman, M. L. Knotek, Phys. Rev. B, 18 (1978) 6531.

[20] Y. Miyamoto, N. Jinbo, H. Nakamura, A. Rubio and D. Tomanek, Phys. Rev. B 70 (2004) 233408.

[21] F. Mauri, R. Car, Phys. Rev. Lett. 75 (1995) 3166.

[22] S. Tanaka, Y. Kayanuma, Solid State Commun. 100 (1996) 77.

[23] K. A. Mader, S. Baroni, Phys. Rev. B, 55 (1997) 9649.

[24] K. Maeda, S. Liang, Y. Harada, Y. Kitajima, S. Shin, and Y. Mera, Proc. SPIE 6586 Damage to VUV, EUV, and X-ray Optics (2007) 65860C-1-10.

[25] A. Kuznetsova, I. Popova, J. T. Yates Jr., M. J. Bronikowski, C.B. Huffman, J. Liu, R. E. Smalley, H. H. Hwu, J. G. Chen, J. Am. Chem. Soc. 123 (2001) 10699.

[26] C. S. Lee, J.-K. Shin, K. Y. Eun, K.-R. Lee, K. H. Yoon, J. Appl. Phys. 95 (2004) 4829.

[27] G. Comelli, J. Stoehr, C. J. Robinson, W. Jark, Phys. Rev. B 38 (1988) 7511.

[28] S. C. Ray a, K. P. Krishna Kumar, H. M. Tsai, J. W. Chiou, C. W. Pao, W. F. Pong, M.-H. Tsai, B.-H. Wu, C.-R. Sheu, C.-C. Chen, F. C.-N. Hong, H.-H. Cheng, A. Dalakyan, Thin Solid Films 516 (2008) 3374. 
[29] S. Suzuki and Y. Kobayashi, Semiconductor Defect Engineering - Materials, Synthetic Structures and Devices II, (2007), p87, MRS (Warrendale). 


\section{${ }^{*}$ Graphical Abstract (pictogram)}

\section{D/G ratio map}
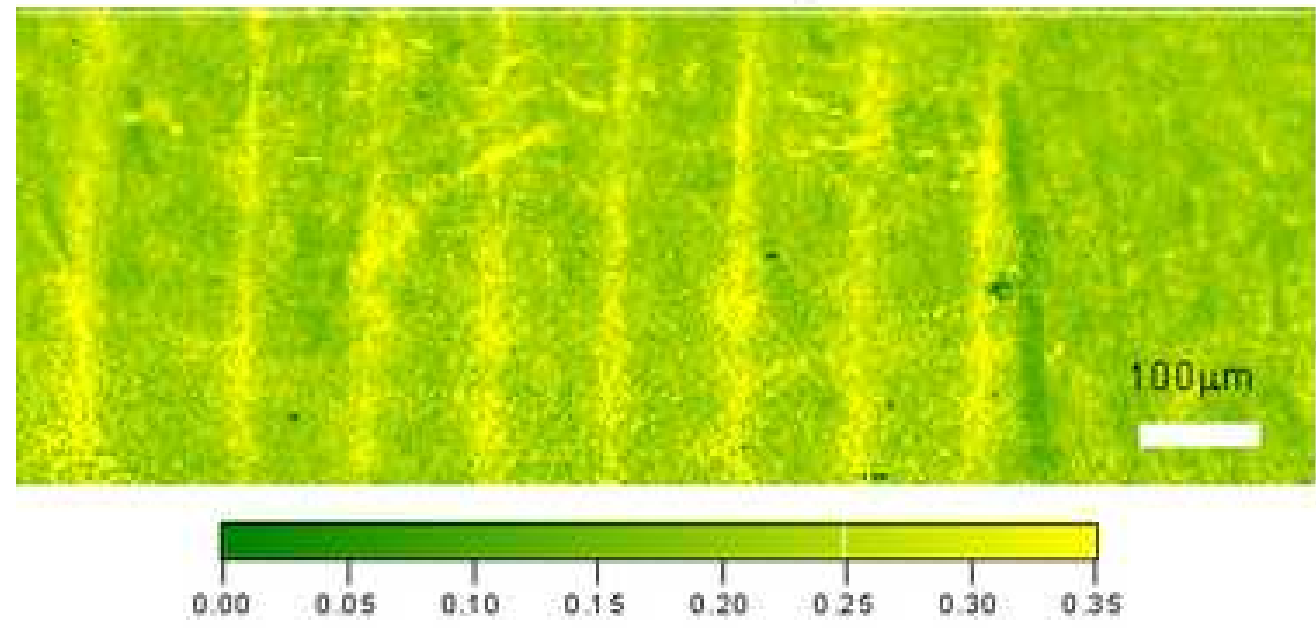

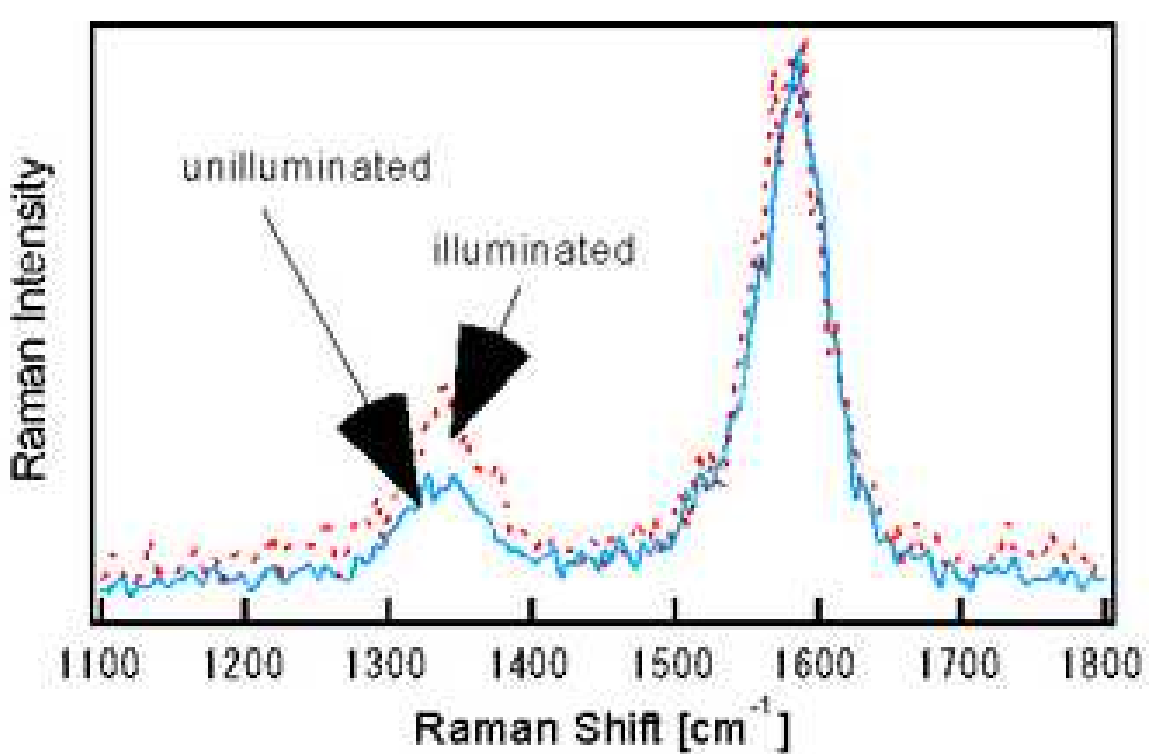


${ }^{*}$ Graphical Abstract (synopsis)

Defects generation in single-walled carbon nanotubes induced by soft X-rays from a synchrotron radiation source was investigated. 